\title{
A Novel Mouse Model of Peritoneal Dialysis: Combination of Uraemia and Long-Term Exposure to PD Fluid
}

\author{
E. Ferrantelli,, ${ }^{1}$ G. Liappas, ${ }^{2}$ E. D. Keuning, ${ }^{1}$ M. Vila Cuenca, ${ }^{1}$ G. González-Mateo, ${ }^{2}$ \\ M. Verkaik, ${ }^{3}$ M. López-Cabrera, ${ }^{2}$ and R. H. J. Beelen ${ }^{1}$ \\ ${ }^{1}$ Department of Molecular Cell Biology and Immunology, VU University Medical Center, Van der Boechorststraat 7, \\ 1081 BT Amsterdam, Netherlands \\ ${ }^{2}$ Centro de Biología Molecular Severo Ochoa, CSIC-UAM, Cantoblanco, 28049 Madrid, Spain \\ ${ }^{3}$ Department of Nephrology, VU University Medical Center, 1007 MB Amsterdam, Netherlands
}

Correspondence should be addressed to E. Ferrantelli; e.ferrantelli@vumc.nl

Received 22 June 2015; Revised 28 September 2015; Accepted 30 September 2015

Academic Editor: Yu-Chang Tyan

Copyright (c) 2015 E. Ferrantelli et al. This is an open access article distributed under the Creative Commons Attribution License, which permits unrestricted use, distribution, and reproduction in any medium, provided the original work is properly cited.

\begin{abstract}
Different animal models for peritoneal dialysis (PD) have been used in the past decades to develop PD fluids compatible with patient life and to identify markers of peritoneal fibrosis and inflammation. Only few of those studies have taken into account the importance of uraemia-induced alterations at both systemic and peritoneal levels. Moreover, some animal studies which have reported about PD in a uremic setting did not always entirely succeed in terms of uraemia establishment and animal survival. In the present study we induced uraemia in the recently established mouse PD exposure model in order to obtain a more clinically relevant mouse model for kidney patients. This new designed model reflected both the slight thickening of peritoneal membrane induced by uraemia and the significant extracellular matrix deposition due to daily PD fluid instillation. In addition the model offers the opportunity to perform long-term exposure to PD fluids, as it is observed in the clinical setting, and gives the advantage to knock out candidate markers for driving peritoneal inflammatory mechanisms.
\end{abstract}

\section{Introduction}

End Stage Renal Disease (ESRD) affects more than 200,000 people in Europe per year and 20,000 of those are peritoneal dialysis (PD) patients. This number is higher in the rest of the world and although in Europe patients undergoing PD are still less than the inmates on hospital-based haemodialysis (HD), this number is expected to increase over the time since $\mathrm{PD}$ succeeds over HD in terms of quality of life [1] and cost effectiveness [2,3]. Moreover, much research on PD has been carried out to improve patient survival and thus qualifies a good alternative for HD treated patients.

Uraemia is represented by accumulation in the body of urea and other organic waste products of metabolism normally filtered out by the kidneys. It can only be treated by replacing kidney function that nowadays, due to the insufficient number of kidneys available worldwide for transplantation, occurs mainly by dialysis.
In the last decade different animal models for PD fluid exposure have been designed and have been mainly performed in rat $[4,5]$. The combining effort of our present groups to study the effect of additions in the rat PD exposure model $[6,7]$ resulted also in the development of the first established mouse PD exposure model published by González-Mateo et al. [8]. PD rodent models have been used to introduce in the market PD fluids more compatible with the patient life and have offered opportunities to study PD fluid additions. Moreover those models allowed identifying important biomarkers driving peritoneal inflammatory mechanisms that occur during long-term exposure to $\mathrm{PD}$ fluids and, as a matter of fact, the failure of the technique itself.

Only few animal studies reported PD in a uremic setting, which is most likely caused by the difficult and delicate procedure needed to induce uraemia, resulting in a low success rate of this technique. 

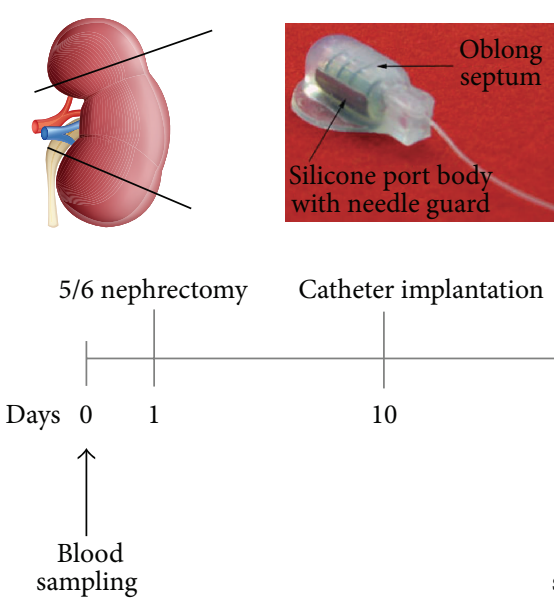

Catheter implantation

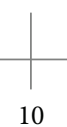

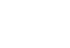

(1)
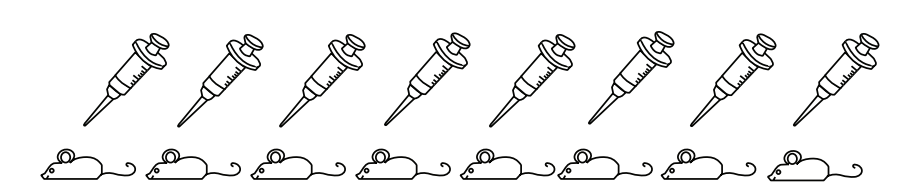

Daily PD fluid instillation
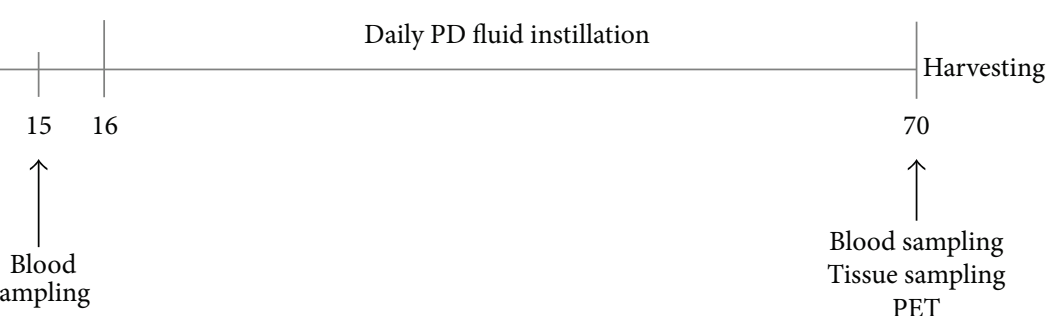

16

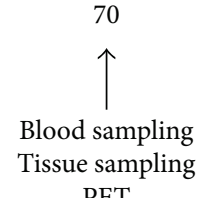

PET

FIGURE 1: Experimental design. 5/6 nephrectomy was executed at day 1 . After a resting period of 10 days a customer made catheter was implanted under the skin at the back side with the tip positioned within the peritoneal membrane. Daily PD fluid instillation was performed from day 16 to day 70 when the mice were sacrificed; PET test and tissue sampling were carried out. Blood withdrawal was performed at days 0,15 , and 70 in order to verify uraemia establishment.

In the present study we wanted to get close to the PD patient clinical condition by combining a long-term exposure model of PD in mouse with uraemia, sine qua non condition for a patient to start PD treatment or a renal replacement therapy in general.

\section{Methods}

2.1. Animals and Experimental Design. The study was performed on 30 female C57BL/6 mice (Harlan CPB, Horst, Netherlands) aged 12-14 weeks, weighing approximately $20 \mathrm{~g}$ at the start of the study. Animals were randomly assigned to the following groups: 10 uremic mice undergoing 5/6 nephrectomy (5/6 NX), 10 uremic PD mice undergoing 5/6 nephrectomy and catheter implantation and exposed to PD fluid (5/6 NX + PD), and 10 healthy controls (control). Mice were housed under standard conditions and were given food and water ad libitum. Health conditions were checked daily. Mice were weighed daily after surgery during a period of 10 days and weekly for the remainder of the experiment. Animals that lost more than $20 \%$ of their body weight or showed abnormal activity were excluded from the experiment. The experimental protocol was approved by the Animal Welfare Committee at the VU University Medical Center, Amsterdam. Experimental design is shown in Figure 1.

2.2. 5/6 Nephrectomy. In order to make mice uremic, 5/6 nephrectomy was performed under isoflurane anaesthesia ( $4 \%$ for induction, $2-3 \%$ for maintenance). $0.05-0.1 \mathrm{mg} / \mathrm{kg}$ of buprenorphine (Temgesic) was injected intramuscularly 1530 minutes preoperatively. The animal was shaved around the abdominal region and was placed on a heating pad. A ventral midline incision was made through the skin followed by an incision along the linea alba. Through the laparotomy the left kidney was released from its capsule by using surgical forceps and wet cotton swabs. At this point the kidney could be easily positioned on top of the peritoneum and was placed on a wound pad. The anterior and posterior $1 / 3$ part of the kidney were impaired by using a monopolar electric blade. The remaining functional $1 / 3$ of the left kidney was placed back into its original position in the abdominal cavity. Following the same procedure, also the right kidney was removed from the abdominal cavity and released from the capsule. A total ligation with insoluble suture was applied, which included the kidney vein, artery, and urethra. After ligation, the right kidney was completely removed. This procedure resulted in a reduced kidney function by $5 / 6$ th of its original function. The main steps of the procedure are shown in Figure 2.

2.3. Catheter Implantation. Customized mouse catheter (MMP-4S-061108A, Access Technologies, Ridgeway, USA) was implanted at day 10 under isoflurane anaesthesia ( $4 \%$ for induction, 2-3\% for maintenance). An incision in the skin was made, skin was separated from muscle layer, and a small hole was made in the lateral side of the abdomen by means of a needle. The catheter was implanted under the skin with the mouse-o-port positioned subcutaneously at the back-right side and the tip within the peritoneal cavity through the hole previously made. The whole procedure is described by González-Mateo et al. [8].

2.4. Peritoneal Exposure Model. Instillation of $2 \mathrm{~mL}$ of peritoneal dialysis fluid (PDF) (Dianeal 3, 86\%, Baxter, USA) was performed daily during a period of 8 weeks via the previously implanted mouse-o-port connected to the peritoneal cavity via the catheter. First injection was conducted after a resting period of 7 days from surgery to allow the operational wound's healing process around the catheter and avoid leakage of fluid outside the peritoneal cavity.

2.5. Serum Analysis. $200 \mu \mathrm{L}$ of blood was drawn via facial vein puncture at days 0 and 15 (resp., before the nephrectomy and the first injection of PD fluid) and at day 70 (end point). 


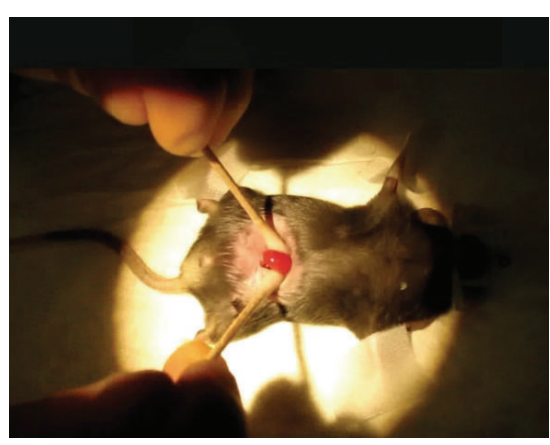

(a)

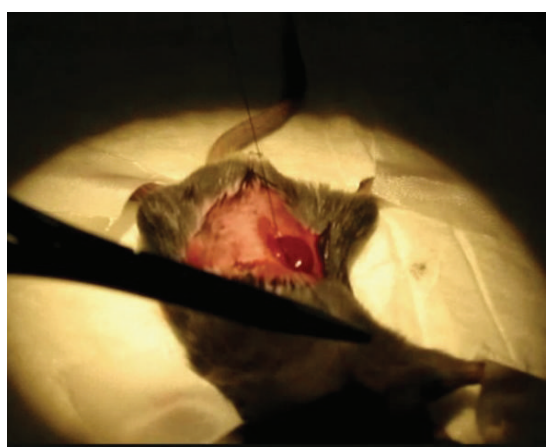

(d)

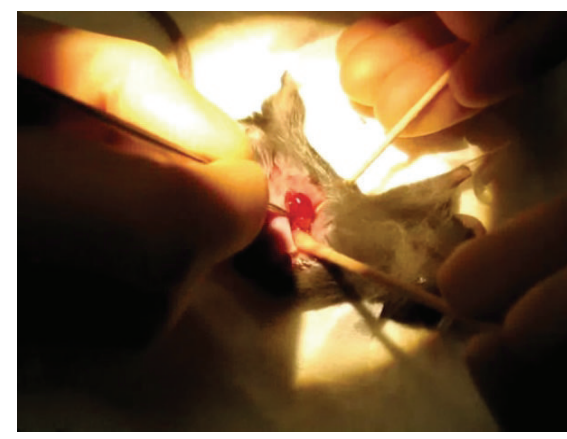

(b)

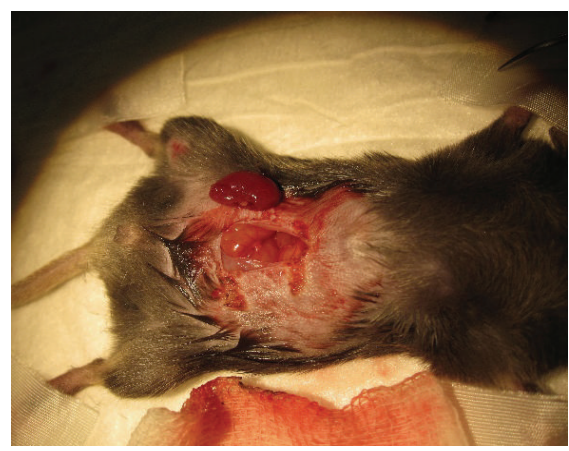

(e)

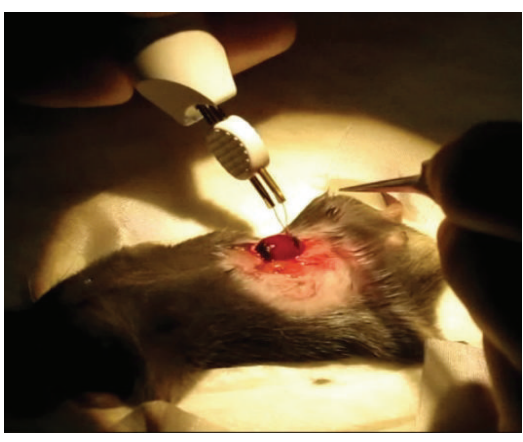

(c)

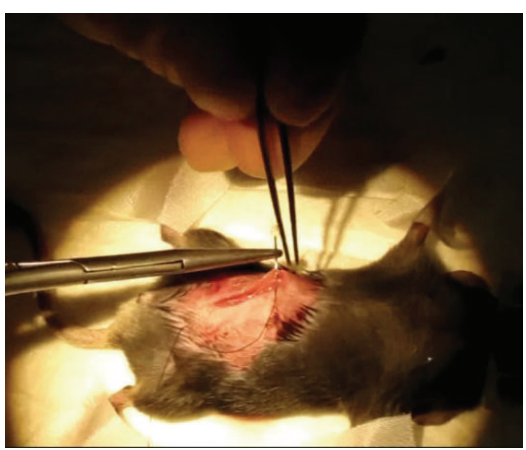

(f)

FIGURE 2: 5/6 nephrectomy: surgical procedure. (a) Through the laparotomy the left kidney was released from its capsule by using surgical forceps and wet cotton swabs. (b) Left kidney was released from the capsule. (c) A monopolar electric blade was used to impair the anterior and posterior 1/3 part of the kidney. (d) A total ligation with insoluble suture was applied, which included the kidney vein, artery, and urethra. (e) Right kidney was totally removed from the body. (f) Both muscle layer and skin were closed by continuous sutures.

At all the time points, serum samples were analysed for urea and creatinine levels. For determination of urea levels a kinetic test with urease and glutamate dehydrogenase was used. Creatinine levels were detected by indirect immunofluorescence assay. Measurements were performed by using spectrophotometer Cobas8000 (c702), Roche Diagnostics.

2.6. Immunostaining for Peritoneal Thickness. Parietal peritoneal biopsies were collected from the opposite side from the catheter installation. Biopsies were fixed in Bouin's solution, embedded in paraffin, cut into $5 \mu \mathrm{m}$ sections, and stained with Masson's trichrome. Peritoneal membrane thickness was determined using light microscopy (Leica CTR6000, with Leica Microsystems LAS-AF6000). Photographs were made using Olympus BX41 clinical microscope and Olympus DP20 digital camera using cell acquisition software. Peritoneal thickness of each animal was calculated by the median of measurement taken every $50 \mu \mathrm{m}$ from one side to the other of the tissue sample.

2.7. Statistical Analysis. Data were analysed using GraphPad Prism software (La Jolla, CA). Statistical analysis was performed using one-way ANOVA test to compare the groups. A $P$ value $<0.05$ was considered statistically significant. Urea and creatinine data were shown as means \pm SD. Thickness is represented in boxplots.

\section{Results}

In order to mimic in mice the clinical situations of peritoneal dialysis patients, uraemia was induced by performing $5 / 6$ nephrectomy. This 5/6 nephrectomy surgery resulted in a functional kidney capacity $1 / 6$ th of the original kidney volume. 15 days after surgery a significant increase of serum urea levels was already seen (data not shown), which was maintained throughout the study period. At the end of the study the nephrectomized mice showed an almost twofold increase in both urea $(15 \pm 2.71$ versus $8.32 \pm 2.38 ; P<0.01)$ and creatinine serum levels $(70.80 \pm 40.00$ versus $33.50 \pm$ 6.95) when compared to the healthy control group (Figures $3(\mathrm{a})$ and 3(b)), indicative for a uremic status. Throughout the experiment nephrectomized mice were in good health as indicated by increasing body weight during the study period, which was comparable between all three groups. As expected, all nephrectomized mice showed a drop in body weight within the first week after surgery but recovered the following week (Figure 3(c)).

The PDF-treated group showed a significant increase in peritoneal thickness compared to both the 5/6 NX and the control groups (Figure 4; $41.14 \pm 5.04$ versus $26.71 \pm 3.59$ versus $13.00 \pm 3.16 ; P<0.001)$. This demonstrates that chronic instillation of PDF in our mouse model caused peritoneal thickening and inflammation of the submesothelial compact zone comparable with the clinic situation of a patient 


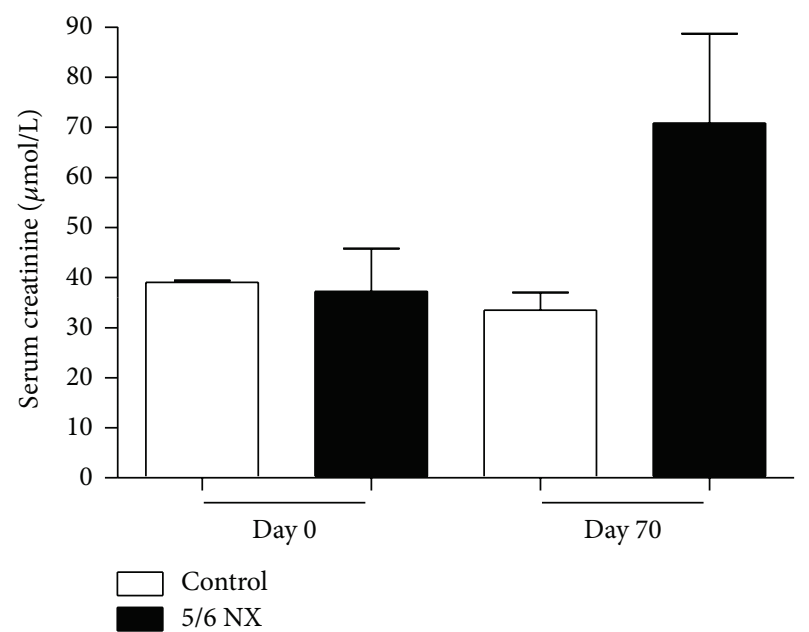

(a) Serum creatinine

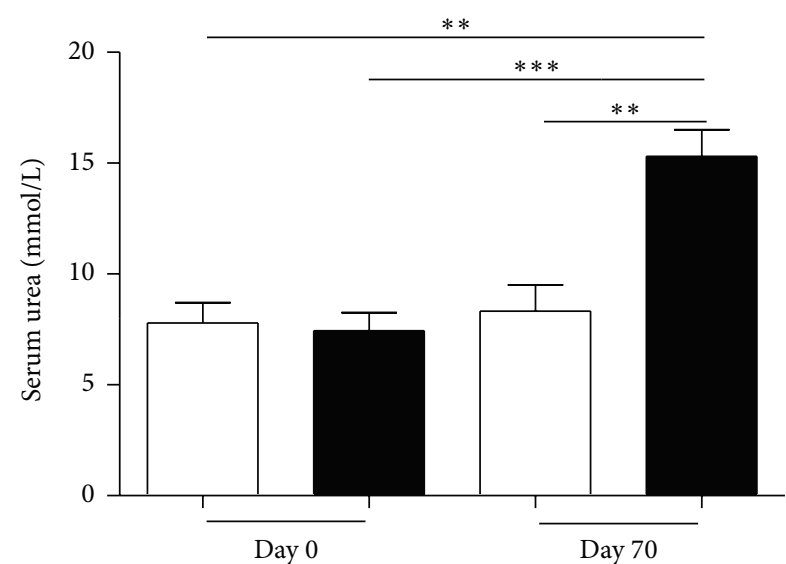

Control $5 / 6 \mathrm{NX}$

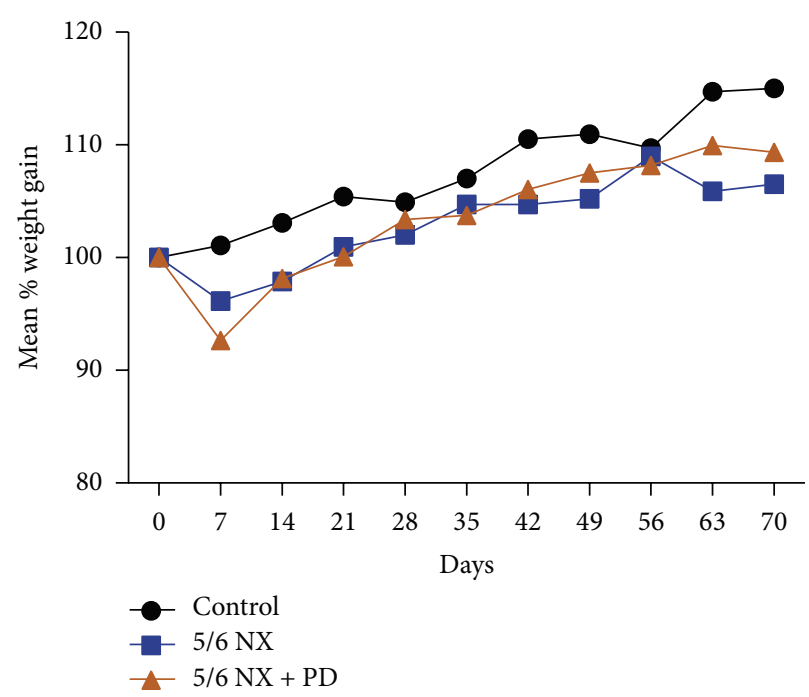

(c) Body weight

Figure 3: ( $\mathrm{a}$ and b) Blood chemistries at days 0 and 70, respectively, before and after induction of uraemia with 5/6 nephrectomy. (a) Serum levels of creatinine. Time 0: $37.20 \pm 19.165 / 6 \mathrm{NX}$ versus $39.00 \pm 0.82$ control; time 70: $70.80 \pm 40.005 / 6 \mathrm{NX}$ versus $33.50 \pm 6.95$ control. (b) Serum levels of urea: white bars indicate healthy controls; black bars indicate mice undergoing $5 / 6$ nephrectomy. $P$ values $* *<0.01$, $* * *<0.001$. (c) Mean increases in body weight during uraemia induction. Values are expressed as percent of starting body weight. Black line indicates healthy controls, blue line indicates uremic group, and red line indicates uremic group undergoing PD.

undergoing PD. Moreover, the peritoneal thickening already found in the nephrectomized group showed that uraemia cannot be omitted in a model of PD.

\section{Discussion}

Uraemia is the terminal clinical manifestation of kidney failure and it represents the main reason for a patient to be introduced to a dialysis treatment. Systemic changes that occur in uremic patients such as the significant increase in advanced glycation end products (AGEs), nitric oxide synthase (NOS), Tumor Necrosis Factor Alpha (TNF $\alpha$ ), and Vascular Endothelial Growth Factor (VEGF) levels, but also hyperosmolarity and blood pressure itself, may influence peritoneal permeability and cause thickening of the extracellular matrix and mild vasculopathy $[9,10]$. These alterations induce peritoneal damage and may therefore complicate the PD procedure in patients.

Peritoneal damage in PD patients mainly depends on the balance between chronic damage caused by bioincompatibility of the PD fluids currently available in the market and repair mechanisms. Over the years different animal models of PD have been used to research into the use of different peritoneal dialysis fluids [11-14] and addition of substances to the dialysis fluid and treatment $[6,15]$.

Animal studies have shown an independent contribution of uraemia to modulating inflammatory events that alter the function of the peritoneal membrane although these events 


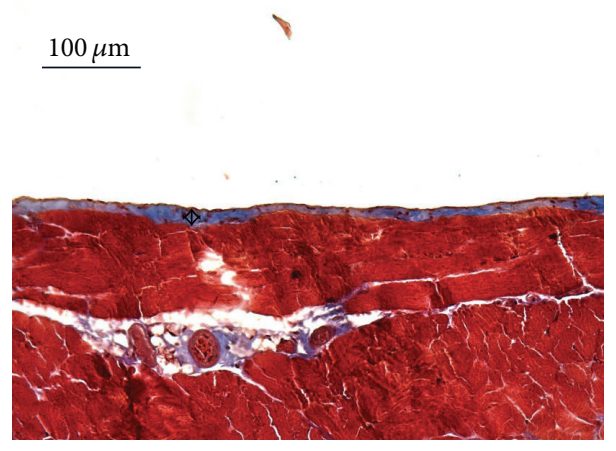

(a) Control

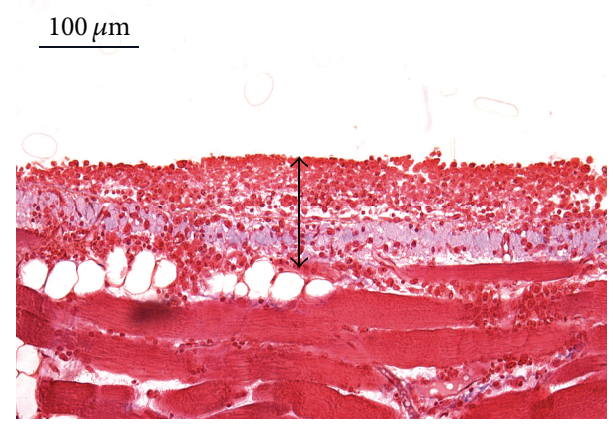

(c) Dianeal $(5 / 6 \mathrm{NX})$

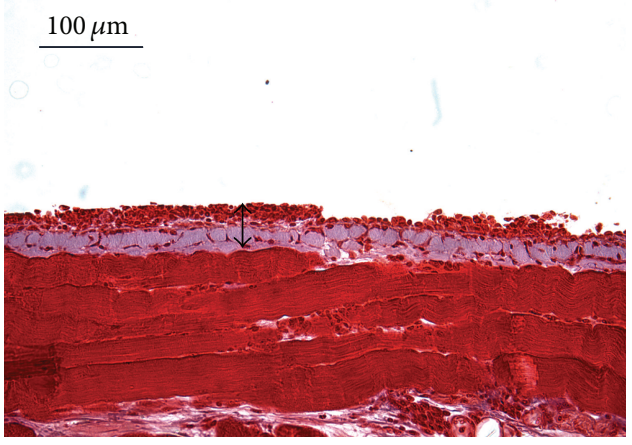

(b) $5 / 6 \mathrm{NX}$

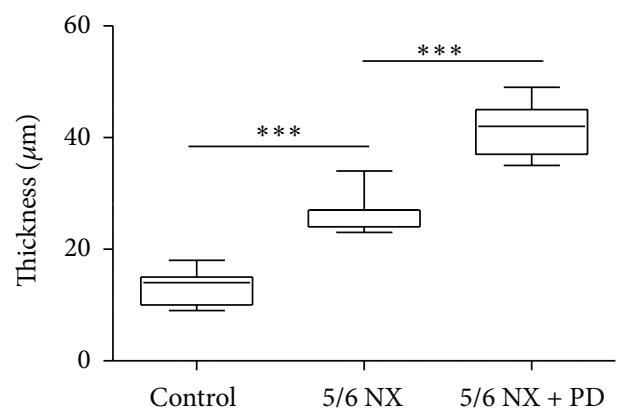

(d) Thickness

FIGURE 4: Effect of uraemia and PD fluid exposure on the parietal peritoneal thickness. (a-d) Masson's trichrome staining of parietal peritoneum showed a slight and a high increase of extracellular matrix deposition in mice undergoing 5/6 nephrectomy, respectively, exposed (c) or not (b) to standard PD fluid. Magnification $\times 20$. (Control: $13.00 \pm 3.16$; 5/6 NX: 26.71 \pm 3.59; 5/6 NX + PD: $41.14 \pm 5.04$.) $P$ values $* * * 0.001$.

are often obscured by the effects of PD fluid induced injury [11]. Nevertheless, in anephric rats undergoing continuous ambulatory peritoneal dialysis (CAPD) uraemia modifies the permeability of peritoneum to both water and solutes [16]. In rat models exposed to PD fluid uraemia contributed to ultrafiltration failure, leading to angiogenesis and causing an increment in omental mast cells number [13].

Uraemia-induced alterations at both systemic and peritoneal level should not be underestimated and experimental animal models of renal disease should take into account the important effect of uraemia. Although the study of the peritoneal membrane alterations promoted by the PD fluids alone, avoiding the effect of other variables, is a very helpful model, it could be very interesting for several approaches to analyse the combined effect of PD exposure and uraemia.

Despite that, only few studies report on PD in uremic animals. The procedure to obtain uraemia in animals indeed is not so easy and many models still show high percentage of drop-out and low rate of success especially when combined with long-term exposure to PD fluids. In rabbit models partial nephrectomy (total removal of one kidney and 5/6 nephrectomy of the other one) has been performed but only moderate uraemia has been reached [17]. More studies have been performed on rats: bilateral nephrectomy caused acute uraemia and, on the other hand, the removal of only one kidney did not induce significant changes in urea and creatinine blood levels. Moreover, animals were experiencing diarrhea and loss of appetite and body weight [16].

Our group also had previously developed a uremic rat PD model consisting of total removal of the left kidney and double artery ligation of the right one (5/6 nephrectomy). In this model both urea and creatinine serum levels were shown to be threefold increased after only three weeks from the nephrectomy and kept stable during five weeks of exposure to PD fluid (Ferrantelli et al., pending revision). Although this model allowed us to obtain important striking results regarding the protective effect of some additive to the PD fluid, the percentage of drop-out was still too high. Unfortunately, 5/6 nephrectomy itself in rats caused $20 \%$ of drop-out, with even higher drop-out when technical failure due to omentum wrapping of the catheter during the PD treatment was taken into account.

In this study we present a novel animal model where both uraemia and daily PD treatment were combined in mouse. As a consequence of the 5/6 nephrectomy only $1 / 6$ th of the total kidney volume is preserved and remains functional during the whole period of daily peritoneal fluid administration.

This model fulfils the need to reduce the percentage of drop-out due to both nephrectomy and PD treatment. Dropout caused by $5 / 6$ nephrectomy was decreased from $20 \%$ in the rat model to less than 5\% in the mouse model. Moreover, this model circumvents PD treatment-related loss of animals 
such as omentum overgrowth and wrapping of the catheter (drop-out due to catheter obstruction is about $50 \%$ in the rat model) [5].

Taken together, this new technique reduces the number of animals needed for research and enables extending time of exposure to PDFs. Indeed, daily treatment of this mouse model with PDF for 8 weeks was well tolerated and might be extended for a longer period. Our consideration is based on the evidences that the animals were healthy during and till the end of the experiment (no evidences of discomfort and no loss of body weight or decrease of appetite) and the serum levels of both creatinine and urea were twofold increased compared to the controls but still within acceptable levels.

The PD mouse model represents a gold standard procedure [8] and many experiments based on it have been performed in the last years in order to study Epithelialto-Mesenchymal Transition (EMT) and the involvement of Th17 cells in fibrotic mechanisms occurring during long-term exposure to PDF $[18,19]$.

In our study we wanted to propose the PD mouse model within a uremic setting in order to mimic as closely as possible the situation in a patient with chronic kidney failure undergoing PD. In addition our mouse model will give the advantage to knock out genes playing a crucial role in the peritoneal inflammatory mechanism that occurs during PD and will open opportunities to study pathways involved in fibrosis, since much more reagents are available in mice.

\section{Conclusions}

Besides the PD-related effects on fibrosis in mice, we showed that uraemia also affects this phenomenon, which is in accordance with patient studies.

To mimic this clinical setting, we developed a mouse model with important features observed in renal failure patients. Indeed this new mouse model gives the opportunity to study PD in concomitance with peritoneal and systemic changes caused by uraemia, often not taken into account in animal studies. Importantly, long-term in vivo experiments can be performed in this model, eventually resulting in less harmful effects of biocompatible PDFs as well as PDFs supplemented with protective additives, both favorable for patients.

\section{Conflict of Interests}

The authors declare that there is no conflict of interests regarding the publication of this paper.

\section{Acknowledgment}

EuTRiPD, Marie Curie actions, supported this work, Grant no. ITN 287813.

\section{References}

[1] A. Berger, J. Edelsberg, G. W. Inglese, S. K. Bhattacharyya, and G. Oster, "Cost comparison of peritoneal dialysis versus hemodialysis in end-stage renal disease," American Journal of Managed Care, vol. 15, no. 8, pp. 509-518, 2009.

[2] A. J. Collins, R. N. Foley, C. Herzog et al., "Excerpts from the US renal data system 2009 annual data report," American Journal of Kidney Diseases, vol. 55, no. 1, supplement 1, pp. S1-S420, A6A7, 2010.

[3] D. C. Mendelssohn, S. K. Mujais, S. D. Soroka et al., "A prospective evaluation of renal replacement therapy modality eligibility," Nephrology Dialysis Transplantation, vol. 24, no. 2, pp. 555-561, 2009.

[4] N. Lameire, W. Van Biesen, M. Van Landschoot et al., "Experimental models in peritoneal dialysis: a European experience," Kidney International, vol. 54, no. 6, pp. 2194-2206, 1998.

[5] R. H. J. Beelen, L. H. P. Hekking, M. Zareie, and J. van den Born, "Rat models in peritoneal dialysis," Nephrology Dialysis Transplantation, vol. 16, no. 3, pp. 672-674, 2001.

[6] J. Loureiro, M. Schilte, A. Aguilera et al., "BMP-7 blocks mesenchymal conversion of mesothelial cells and prevents peritoneal damage induced by dialysis fluid exposure," Nephrology Dialysis Transplantation, vol. 25, no. 4, pp. 1098-1108, 2010.

[7] M. N. Schilte, J. W. A. M. Celie, P. M. Ter Wee, R. H. J. Beelen, and J. van den Born, "Factors contributing to peritoneal tissue remodeling in peritoneal dialysis," Peritoneal Dialysis International, vol. 29, no. 6, pp. 605-617, 2009.

[8] G. T. González-Mateo, J. Loureiro, J. A. Jiménez-Hefferman et al., "Chronic exposure of mouse peritoneum to peritoneal dialysis fluid: structural and functional alterations of the peritoneal membrane," Peritoneal Dialysis International, vol. 29, no. 2, pp. 227-230, 2009.

[9] J. D. Williams, K. J. Craig, N. Topley et al., "Morphologic changes in the peritoneal membrane of patients with renal disease," Journal of the American Society of Nephrology, vol. 13, no. 2, pp. 470-479, 2002.

[10] S. Combet, M.-L. Ferrier, M. Van Landschoot et al., "Chronic uremia induces permeability changes, increased nitric oxide synthase expression, and structural modifications in the peritoneum," Journal of the American Society of Nephrology, vol. 12, no. 10, pp. 2146-2157, 2001.

[11] M. Zareie, A. A. van Lambalgen, P. M. ter Wee et al., "Better preservation of the peritoneum in rats exposed to amino acidbased peritoneal dialysis fluid," Peritoneal Dialysis International, vol. 25, no. 1, pp. 58-67, 2005.

[12] M. Zareie, L. H. P. Hekking, A. G. A. Welten et al., "Contribution of lactate buffer, glucose and glucose degradation products to peritoneal injury in vivo," Nephrology Dialysis Transplantation, vol. 18, no. 12, pp. 2629-2637, 2003.

[13] M. N. Schilte, P. Fabbrini, P. M. Ter Wee et al., "Peritoneal dialysis fluid bioincompatibility and new vessel formation promote leukocyte-endothelium interactions in a chronic rat model for peritoneal dialysis," Microcirculation, vol. 17, no. 4, pp. 271-280, 2010.

[14] L. H. Hekking, M. C. Aalders, E. Van Gelderop et al., "Effect of peritoneal dialysis fluid measured in vivo in a rat-model of continuous peritoneal dialysis," Advances in Peritoneal Dialysis, vol. 14, pp. 14-18, 1998.

[15] P. Fabbrini, M. N. Schilte, M. Zareie et al., "Celecoxib treatment reduces peritoneal fibrosis and angiogenesis and prevents ultrafiltration failure in experimental peritoneal dialysis," Nephrology Dialysis Transplantation, vol. 24, no. 12, pp. 3669-3676, 2009.

[16] K. Pawlaczyk, M. Kuzlan-Pawlaczyk, K. Wieczorowska-Tobis, A. Polubinska, A. Breborowicz, and D. Oreopoulos, "Evaluation 
of the effect of uremia on peritoneal permeability in an experimental model of continuous ambulatory peritoneal dialysis in anephric rats," Advances in Peritoneal Dialysis-Conference on Peritoneal Dialysis, vol. 15, pp. 32-35, 1999.

[17] L. Gotloib, P. Crassweller, H. Rodella et al., "Experimental model for studies of continuous peritoneal'dialysis in uremic rabbits," Nephron, vol. 31, no. 3, pp. 254-259, 1982.

[18] G. T. González-Mateo, V. Fernández-Míllara, T. Bellón et al., "paricalcitol reduces peritoneal fibrosis in mice through the activation of regulatory $\mathrm{T}$ cells and reduction in IL-17 production," PLoS ONE, vol. 9, no. 10, Article ID e108477, 2014.

[19] R. Rodrigues-Díez, L. S. Aroeira, M. Orejudo et al., "IL-17A is a novel player in dialysis-induced peritoneal damage," Kidney International, vol. 86, no. 2, pp. 303-315, 2014. 


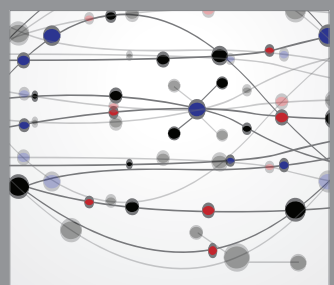

The Scientific World Journal
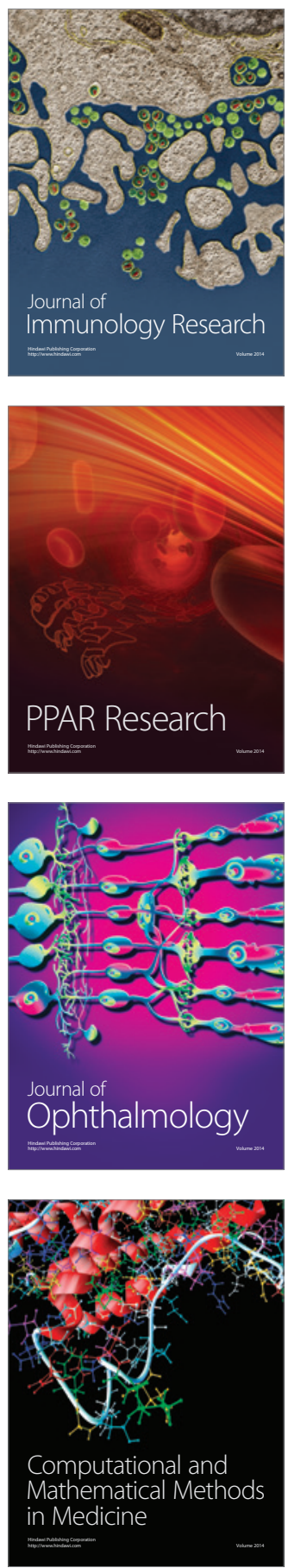

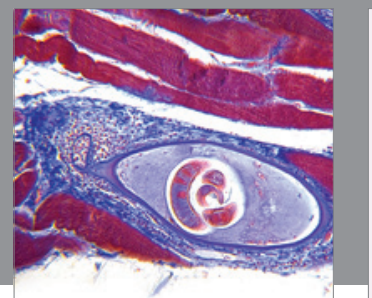

Gastroenterology

Research and Practice
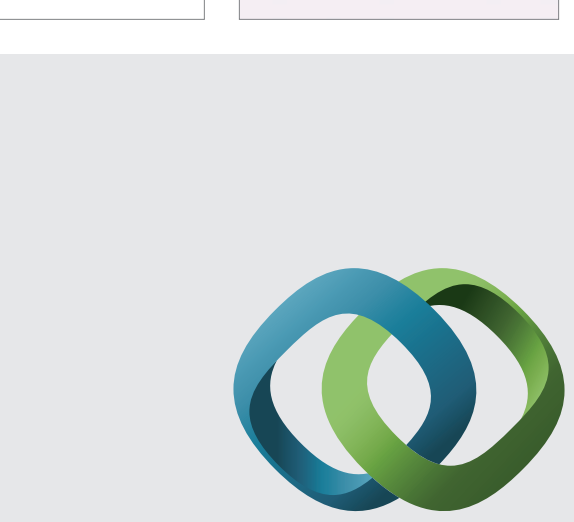

\section{Hindawi}

Submit your manuscripts at

http://www.hindawi.com
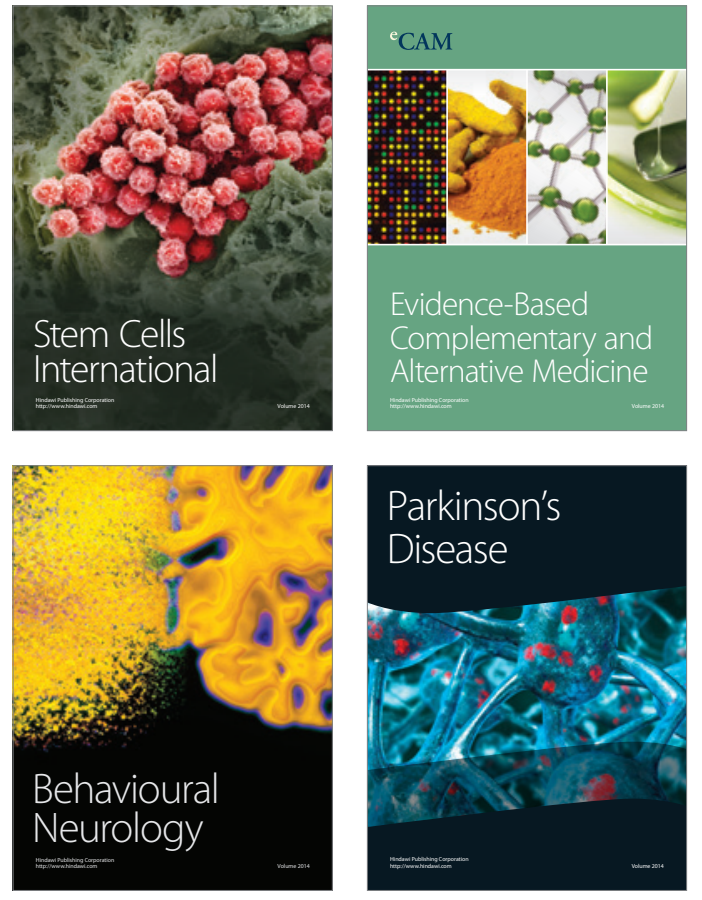
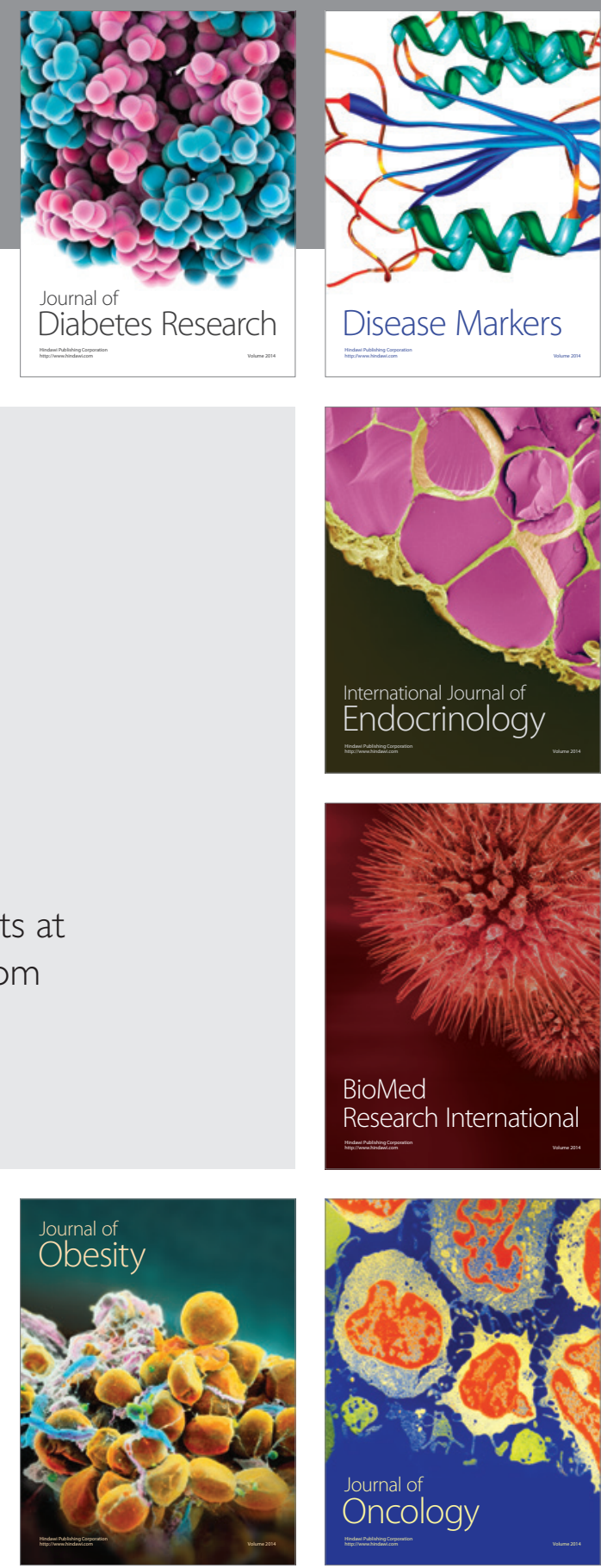

Disease Markers
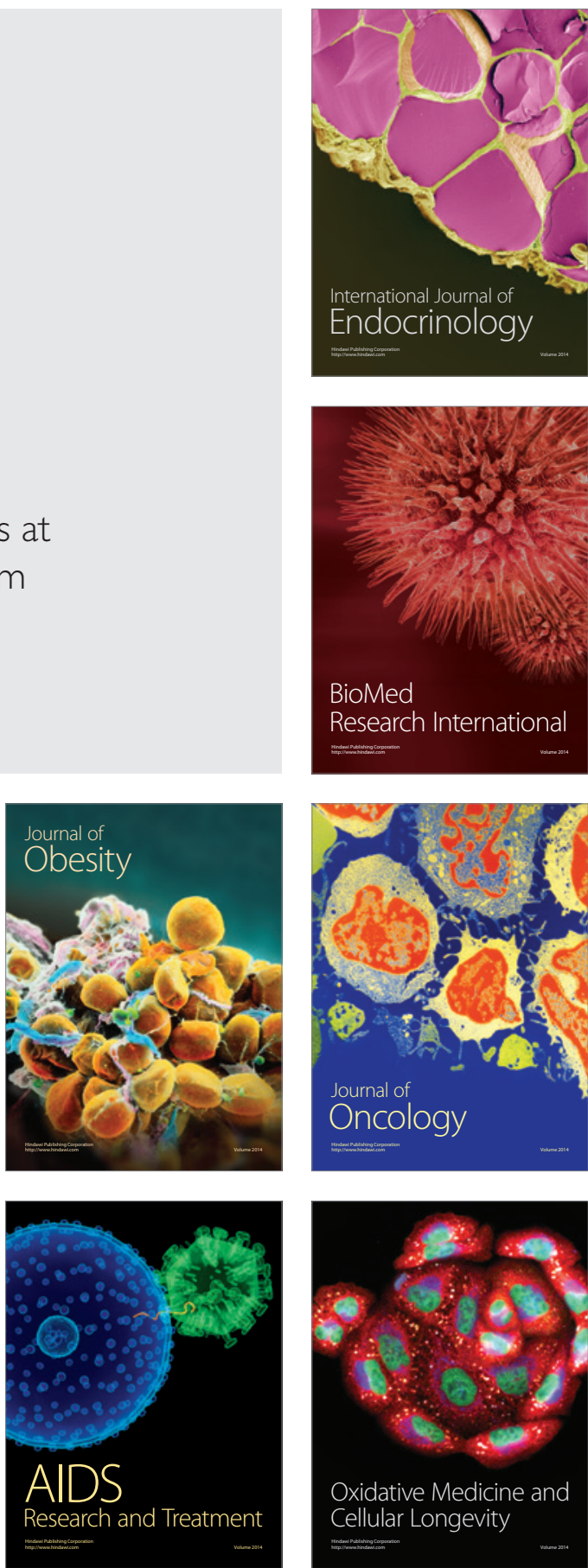